\title{
CLINICAL AND RADIOGRAPHIC ASSESSMENT OF DIFFERENT OCCLUSAL SCHEMES IN “ALL ON 4" CONCEPT
}

\author{
Noha H. Nawar * and Yasmine G. Thabet ${ }^{* *}$
}

\begin{abstract}
Objective: The success of dental implants depends on accurate planning of the occlusal scheme enrolled in the overlying prosthesis, so the present study was conducted to evaluate the effect of two different occlusal schemes on the rate of supporting bone resorption in All on 4 mandibular implant retained overdentures constructed with two occlusal schemes, lingualized and group function. Clinical occlusal analysis was also done using digital T-scan to analyze the occlusion and disocclusion timings.
\end{abstract}

Materials and Methods: Fourteen edentulous male patients were selected according to certain criteria, patients had guided surgical installation of four implants following "All on 4 "concept and were randomly allocated into two equal groups, Group I:rehabilitated with provisional fixed detachable prosthesis following the group function occlusal concept and Group II: rehabilitated with provisional fixed detachable prosthesis following the lingualized occlusal concept. The implants in the two groups were monitored using cone beam computed tomography at loading, 6 months and one year later. Digital occlusal analysis was performed using the T-scan at loading.

Results: The results of the current study showed statistically significant less bone height changes values in group I in comparison to group II as per the radiographic outcomes while the occlusal analysis showed statistically significant increase in disocclusion time (in seconds)in group I.

Conclusion: Lingualized occlusal scheme showed less stresses transmitted to dental implants and their supporting structures as reflected in decreased periimplant bone loss.

KEY WORDS: All on four, occlusion, group function, lingualized occlusion.

\section{INTRODUCTION}

Recently, dental implants have become a very popular solution for edentulism due to several factors. The leap in technology of manufacturing the implants with different surface treatments, besides the increased awareness of the patients contributed to the prevalence of dental implants. In atrophied edentulous jaws, implant supported

*Associate Professor of Removable Prosthodontics, Faculty of Dentistry, Ain Shams University.

**Lecturer of Removable Prosthodontics, Faculty of Dentistry Ain Shams University. 
prosthetic treatment is quiet challenging without complex procedures such as nerve repositioning and grafting in the posterior regions of the maxilla and mandible. Another solution for such situations is the "All-on-4" concept. ${ }^{(1-2)}$

The "All on-4" concept can be an alternative and potential treatment especially in atrophic edentulous jaws. This can be achieved by placing four implants, two straight implants anteriorly and two tilted ones with an angle of $30-45^{\circ}$ in the posterior region. This improves anchorage of the implants since they engage cortical bone from the anterior section of the maxillary sinus and the nasal passage (nostril) and the inter-foraminal region in the mandible. Reconstructive surgeries or bone graft procedures are avoided thus it is less invasive and financially competitive. ${ }^{(3-8)}$

Some authors advocated implant splinting by bar in case of increasing angulation of distal implant more than 30 degree $^{(9)}$

In the "All on-4" treatment modality, implants are splinted by means of a metal super-structure. Tilting of the posterior implants allows the screws to emerge in the second premolars, or first molars regions, thus reducing or avoiding cantilevers and subjecting the dentures to lesser mechanical stresses. Moreover, tilting of the implants increases the inter-implant distance (A-P spread) and preserves anatomical structures yet, it facilitates the use of longer implants. ${ }^{(5-12)}$

The selection of an occlusal scheme for implantsupported prosthesis is often controversial. The occlusal scheme should minimize loads at the bone/ implant interface and implant prostheses, thus maintaining implant loads within the physiological limits of the stomatognathic system and maximizing the longevity of the implants and prosthesis. ${ }^{(12)}$

Almost all occlusal concepts are based on those found in the natural dentitions which are modified to coordinate with the implant support systems.
Thus, the occlusion follows mechanical principles, to direct the forces towards the long axis of the implants, and minimize off-centered forces. ${ }^{(13,15)}$

Balanced occlusion maintains simultaneous contact between maxillary and mandibular teeth in centric and eccentric positions.. It is advocated to avoid denture base displacement during the final gliding occlusal phase of mastication following bolus reduction. Lingualized occlusion can be utilized to facilitate bilateral balance. When a single complete overdenture opposes natural dentition, balance may be practically difficult to achieve. Attempts to achieve at least three points balance during lateral and protrusive excursions should be done. ${ }^{(16)}$

Group Function occlusion is considered as unilateral balanced occlusion. It is utilized on the occlusal surfaces of teeth on one side of the arch, where they occlude simultaneously with a smooth uninterrupted glide. Thus the occlusal load is distributed on the working side, while teeth on the non-working side are not subjected to destructive loads. A study reported that, group function occlusion protects the centric holding cusps from excessive wear thus it helps in maintaining the occlusion. ${ }^{(17,18)}$

The T-Scan III system was developed by Tekscan (Boston, MA, USA) where computers are utilized to analyze dynamic occlusion. Both qualitative and quantitative occlusal analyses are performed simultaneously and accurately. It analyzes the distribution of occlusal forces in the anterior region, between the molars, and between the right and left sides of the dental arch. In addition, it can detect the sequence of contact between opposing teeth and quantitative changes in relation to time. Moreover, it locates excessive occlusal forces, and registers the amount of time from early contact to equilibrium of occlusal forces. ${ }^{(19,20)}$

Many implant failures were attributed to improper occlusal designing which concentrated 
stresses in the bone and lead to rapid bone resorption. The goal of any prosthetic treatment must include establishment of a functional occlusion. Hence, this study was conducted to evaluate the rate of supporting bone resorption as well as the occlusal force analysis in all on 4 implant overdenture in edentulous mandible constructed with two occlusal schemes, balanced and group function, using cone beam $\mathrm{CT}$ and $\mathrm{T}$ scan.

\section{MATERIALS AND METHODS}

\section{Participants}

Fourteen male edentulous patients aged between 50 and 60 years old were selected from the out-patient clinic of the Prosthodontic Department, Faculty of Dentistry, Ain Shams University. After the patients were informed about the line of treatment and the need for frequent recalls throughout the total period of the research, they all signed a written consent. Patients included in the study fulfilled the following criteria: healthy mucosa, complete edentulism for at least 1 year.

All patients had guided surgical installation of four implants following "All on 4 concept".

\section{Patients grouping:}

The patients were randomly assigned using random number generator and checker (www. psychicscience.org/random.aspx) into two equal groups:

Group I: patients were rehabilitated with provisional fixed detachable prosthesis following group function occlusal concept.

Group II: patients were rehabilitated with provisional fixed detachable prosthesis following lingualized occlusal concept.

Methods to achieve the studied occlusal concepts:

All patients received new conventional complete dentures prior to the surgeries which were later modified and used as provisional fixed detachable prosthesis. Dentures were constructed following group function occlusal concept for group I which was achieved by contouring the lingual inclines of the upper buccal cusps to prevent posterior contact from occurring after the lower canine reaches the cusp tip of the upper canine.

Dentures were constructed following the lingualized occlusal scheme for group II where the maxillary palatal cusps contacted the widened central fossae/marginal ridges of the mandibular teeth and one $\mathrm{mm}$ space was present between the buccal cusps in centric occlusion. Working and balancing side contacts occurred on the maxillary palatal cusps as well as 'protrusive contact which was achieved between the maxillary palatal cusps and the lower teeth. In the try-in appointment, the centric relation record was verified and a protrusive record and lateral records were made and the condylar inclinations of the articulator were adjusted accordingly. During the delivery appointment, a remount record was made and the occlusion was refined, in centric and then in eccentric positions.

Patients were allowed at least 2 months to wear the prostheses prior to implant surgery. Double scanning protocol was followed to construct completely limiting CAD-CAM surgical guide following the treatment plan for each group. Gutta Percha markers were inserted in several random positions and in different axial planes on the polished surface of the denture, then a CBCT was made for the patient while wearing the denture. Then another scan was made for the denture only.

The OnDemand 3D computer software was used to select the implant sites according to the optimum site anatomically and functionally, also the locations of the three anchor pins were selected to be on three widely separated positions to be used for fixation of the guide. Once the planning was done, the OnDemand 3D software digitally created the guide from the scanned denture design and was saved as 
an "STL" file (Standard triangular language) to be sent to the Envisiontec digital dental 3D printing machine (Envisiontec, Dearborn, MI, USA) to fabricate a clear surgical guide with a metal sleeves inserted to guide the implant drilling. (Fig 1)

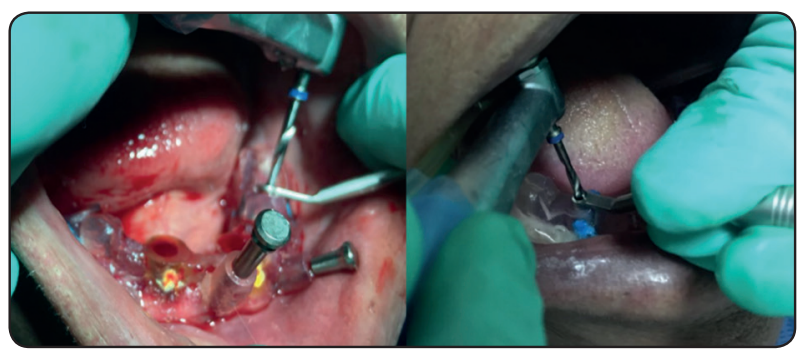

Fig. (1) CAD CAM surgical guide fixed in position.

\section{Surgical and Prosthetic procedure:}

The surgical procedure was the same for the two groups and was performed by the same clinician. It was performed under local anesthesia according to the planning done before. Four implants (Texas MC, Leader, Italy) were installed in interforminal area with the distal implants tilted 30 degrees. Sequential drilling was done after tissue punching and fixation of the surgical guide using fixative pins. Countersinking was performed when needed to create space for the head of the tilted implants. The implant head neck was aimed to be positioned at bone level. The patients were recalled after one week to install the abutments provided that the implant stability reading was more than 50 ISQ (implant stability quotient). Straight and $30^{\circ}$ multiunit abutments with suitable collar heights were placed onto the implants. These are used to achieve the correct access allowing relative parallelism and to allow the rigid prosthesis to be passively seated. Then the provisional complete denture was relived to fix the titanium cylinders of the multiunit abutments using self-cure acrylic resin. (Fig. 2)

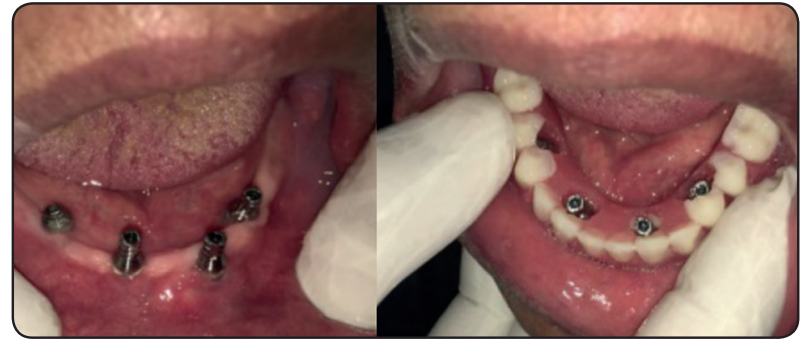

Fig. (2): Multi-unit abutments were placed and the cylindrical titanium parts were fixed to the denture.

\section{Radiographic evaluation:}

Marginal bone height changes around the implants were evaluated using the linear measurement system supplied by the cone beam computed tomography. A ruler in the software was used to measure the bone height from the apex of the implant to the crestal bone in contact with the implant by creating two lines, one tangential to the implants and the other perpendicular to it. (Fig. 3)

The measurements were carried out at the end of each follow up appointment (at insertion, 6, and 12 months post insertion). The marginal bone loss at different intervals was obtained by calculating the difference in bone height at that interval from the base line measurements. Beam hardening correction software "icat" was used to manage the metal artifacts by reconstruction of the image.

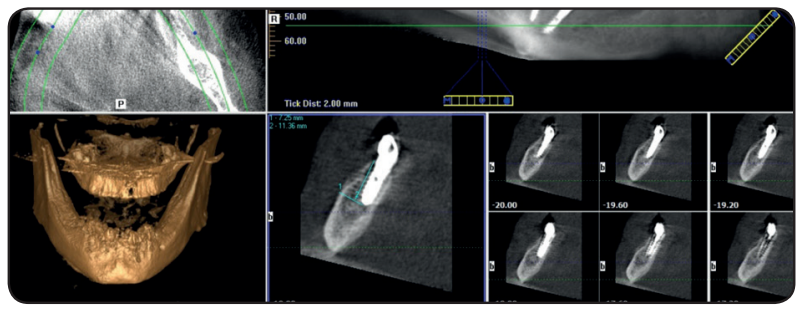

Fig (3): CBCT shows bone height measurements using two lines (tangential and perpendicular to the implants)

\section{Computerized occlusal analysis using the T-Scan III system:}

The patient was seated with his back straight and the sensor was attached to the teeth handle of the T-Scan. Patients were prompted to close 
their mouth to achieve maximum contact between teeth antagonists (maximum intercuspation). Recordings were made in real time at a frequency of $80 \mathrm{~Hz}$ (80 times / sec), and the sequence of tooth contacts over time was measured with an interval of $0,01 \mathrm{sec}$. Resulting movies were processed with the software T-Scan III. The sequence of tooth contact and quantitative changes in relation to time were measured and the amount of time from

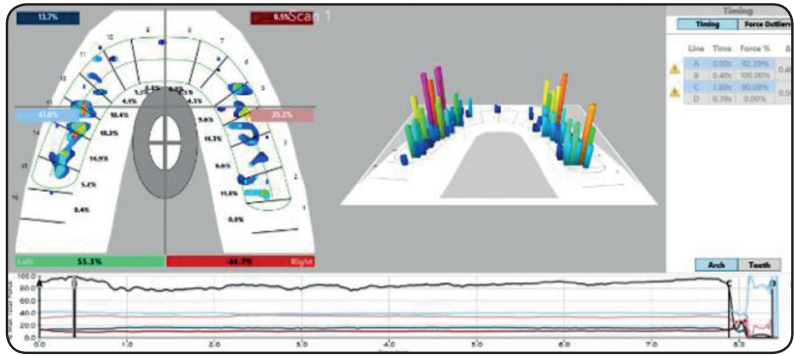

Fig. (5): T scan occlusal analysis showing multiple occlusal contacts in group II.

\section{RESULTS}

Statistical analysis was performed by Microsoft Office 2013 (Excel) and Statistical Package for Social Science (SPSS) version 20. The significant level was set at $\mathrm{P} \leq 0.05$. Kolmogorov-Smirnova and Shapiro-Wilk tests were used to assess data normality. Data were assumed normally distributed. Independent t-test was done to test significance of difference in bone loss around the three implants.

As expressed in table (1), on comparing the measurement of bone loss around the two anterior straight implants obtained from radiographic outcome, there was statistical significant mesial and distal bone loss in group I more than group II during the whole follow-up period.

Fig. (4): T scan screen shot indicating initial contact in group I as indicated by the small blue dot.

TABLE (1): The mean, standard deviation and comparison between decrease in mesial and distal bone height of the straight implant, in group I and II, throughout the whole follow-up period.

\begin{tabular}{|c|c|c|c|c|c|}
\hline & \multicolumn{2}{|c|}{ Group I } & \multicolumn{2}{c|}{ Group II } & \multirow{2}{*}{ P value } \\
\cline { 2 - 5 } & Mean & Std. Deviation & Mean & Std. Deviation & \multirow{2}{*}{$<0.001^{*}$} \\
\hline Mesial 0-6 & 0.39 & 0.09 & 0.16 & 0.04 & $<0.001^{*}$ \\
\hline Distal0-6 & 0.40 & 0.06 & 0.16 & 0.04 & $<0.001^{*}$ \\
\hline Mesial6-12 & 0.35 & 0.05 & 0.23 & 0.058 & $<0.001^{*}$ \\
\hline Distal6-12 & 0.37 & 0.06 & 0.22 & 0.04 & \\
\hline
\end{tabular}


While on comparing the measurement of bone loss around the two distal angled implants obtained from radiographic outcome, there was statistically significant mesial bone loss in group I more than group II during the whole follow-up period (Table 2) .On the other hand, there was no statistical significance in the measurements of distal bone loss.
Regarding occlusion time, there was no statistical difference between the two groups, however regarding disocclusion time group I showed higher values which were statistical significant. Disocclusion time showed higher values compared to occlusion time within the two groups which were statistically significant. (Table 3 )

TABLE (2): The mean, standard deviation and comparison between decrease in mesial and distal bone height of the angled implant, in group I and II, throughout the whole follow-up period.

\begin{tabular}{|c|c|c|c|c|c|}
\hline & \multicolumn{2}{|c|}{ Group 1 } & \multicolumn{2}{c|}{ Group II } & \multirow{2}{*}{ P value } \\
\cline { 2 - 5 } & Mean & Std. Deviation & Mean & Std. Deviation & \\
\hline Mesial 0-6 & 0.42 & 0.07 & 0.19 & 0.03 & $<0.001^{*}$ \\
\hline Distal0-6 & 0.31 & 0.12 & 0.16 & 0.04 & 0.008 \\
\hline Mesial6-12 & 0.40 & 0.07 & 0.20 & 0.04 & $<0.001^{*}$ \\
\hline Distal6-12 & 0.36 & 0.08 & 0.20 & 0.07 & 0.002 \\
\hline
\end{tabular}

TABLE (3): The mean (in seconds), standard deviation, and comparison of occlusion and disocclusion times in the two groups.

\begin{tabular}{|c|c|c|c|c|c|}
\hline \multirow{2}{*}{} & \multicolumn{2}{|c|}{ Group 1 } & \multicolumn{2}{c|}{ Group II } & \multirow{2}{*}{ P value } \\
\cline { 2 - 5 } & Mean & Std. Deviation & Mean & Std. Deviation & \\
\hline Occlusion time & 0.14 & 0.04 & 0.14 & 0.04 & 0.847 \\
\hline Disocclusion time & 0.45 & 0.03 & 0.34 & 0.03 & $<0.001$ \\
\hline P value & \multicolumn{2}{|c|}{$<0.001$} & \multicolumn{2}{c}{$<0.001$} & \\
\hline
\end{tabular}

\section{DISCUSSION}

The clinical and radiographical outcomes of dental implants highly rely on the mechanical setting and the strategy of stress distribution. This issue raised the question of the current study regarding the choice of the suitable occlusal scheme enrolled in such a risky design as the "all on 4" prosthesis.

The T-Scan system was utilized as it provides information that can't be acquired by traditional methods of occlusal measurement and evaluation. Moreover, when repeated measurements for the same subjects were monitored, the results were statistically insignificant, which indicated the validity and reproducibility of the system. ${ }^{(19,20)}$

The basic requirements of the occlusal scheme of an implant-prosthetic superstructure are the establishment of stabilized jaw relationships with maximum intercuspation that are bilaterally identical "in addition to the necessity of freedom in centric". (21)

Simultaneous bilateral contacts on the canine and the posterior teeth and grazing contacts on 
the incisors is recommended by many authors, as this facilitates the wide distribution of the forces transmitted to the supporting implants. It also decreases the disocclusion time which might explain the results of the current study. ${ }^{(16)}$

Lingualized occlusion offers less occlusal interference and surface contacts between the maxillary and mandibular teeth and, consequently, fewer mandibular denture loadings during eccentric movements which could explain the current results.(22-23)

Group function could be defined as multiple contacts between maxillary and mandibular teeth on the working side during lateral movements, where simultaneous contact of several teeth occurs as a group to distribute the occlusal forces. The group function of the teeth on the working side distributes the occlusal load. ${ }^{(22)}$

Group function occlusion was recommended by many authors as the occlusal pattern for prosthetic rehabilitation in older patients. It helps achieve harmonious balance of muscles, temporomandibular joints, and teeth. ${ }^{(18,24)}$ Jemt and Lundiquist ${ }^{(24)}$ stated that chewing pattern may be influenced by the type of occlusion. They found that the angle of departure was steeper than that of approach and these angles were slightly greater with group function. This could justify the increased disocclusion time recorded in overdentures enrolling group function as an occlusal scheme. Moreover, this might cause the increased stresses applied on the straight implants in group I as the working side in group function is responsible for disoccluding the balancing side.

\section{CONCLUSION}

Lingualized occlusal scheme showed less stresses transmitted to dental implants and their supporting structures as reflected in decreased periimplant bone loss.

\section{REFERENCES}

1. Christopher et al: Implant rehabilitation in the edentulous jaw: The "All-on-4® concept" immediate function, Australian dental practice cited, 2012, page 138-48.

2. Singh P., Cranin A.N.: Atlas of oral implantology. Page $275-81.3^{\text {rd }}$ ed 2009

3. Durkan R.1. and Oyar P.: All-On-Four Concept in Implant Dentistry: A Literature Review, J. of Dent. Oral Care Med., 2017: 3 (2): 205-210.

4. Maló P., Rangert B., Nobre M.: "All-on-Four" immediatefunction concept with Brånemark System implants for completely edentulous mandibles: a retrospective clinical study, Clin. Implant Dent Relat Res., 2003, 1:2-9.

5. Maló P., Rangert B., Nobre M.: All-on- $4^{\mathrm{TM}}$ immediatefunction concept with Brånemark System ${ }^{\circledR}$ implants for completely edentulous maxillae: a 1-year retrospective clinical study, Clin. Implant Dent Relat Res. 2005: 7(1): 88-94.

6. Maló P., Nobre Mde A., Petersson U., Wigren S.: A pilot study of complete edentulous rehabilitation with immediate function using a new implant design: case series, Clin. Implant Dent Relat Res. 2006: 8(4):223-32

7. Maló P., de Araujo Nobre M., Lopes A.: The use of computer-guided flapless implant surgery and four implants placed in immediate function to support a fixed denture: preliminary results after a mean follow-up period of thirteen months: J. Prosth. Dent., 2008: 99(3):167.

8. Pomares C.: A retrospective clinical study of edentulous patients rehabilitated according to the 'all on four' or the 'all on six' immediate function concept, Eur. J Oral Implantol., 2009:2(1):55-60.

9. Duyck J,Van Oostcrwyck H,Vander Sloten J.:Magnitude and distribution of occlusal forces on oral implants supporting fixed prosthesis-An in vivo study.Clin Oral implants Res.2000:111:465-475

10. Agliardi E., Panigatti S., Clericò M., Villa C., Malò P.: Immediate rehabilitation of the edentulous jaws with full fixed prostheses supported by four implants: interim results of a single cohort prospective study, Clin. Oral Implants Res, 2010:21(5):459-65.

11. Testori T., Del Fabbro M., Capelli M., Zuffetti F., Francetti L., Weinstein R.L.: Immediate occlusal loading and tilted implants for the rehabilitation of the atrophic edentulous maxilla: 1-year interim results of a multicenter prospective study, Clin. Oral Implants Res, 2008 19(3):227-32. 
12. Verma M., Nanda A., Sood A.: Principles of occlusion in implant dentistry, J. Int Clin Dent Res Organ, 2015:7(1): 27-33

13. Gartner J.L., Mushimoto K., Weber H.P., Nishimura I.: Effect of osseointegrated implants on the coordination of masticatory muscles: A pilot study, J. Prosthet Dent, 2000: 84:185-93.

14. Rangert B., Krogh P.H., Langer B., Van Roekel N.; Bending overload and implant fracture: A retrospective clinical analysis, Int J. of Oral Maxillofac Implants 1995:10: 326-34.

15. Peck C.C.: Biomechanics of occlusion-implications of oral rehabilitation, J Oral Rehab., 2016,43:205-214.

16. V. Rangarajan, P. B. Yogesh, B. Gajapathi, M. Mohamed Ibrahim, R. Ganesh Kumar, Murali Karthik ,Concepts of occlusion in prosthodontics: A literature review, part II The Journal of Indian Prosthodontic Society 2016:16:8-14.

17. Shantanu J., Mohit K., Mukund K., Ramandeep D.: Occlusion and occlusal consideration in implantology, Indian $\mathrm{J}$ of Dental Advancements, 2010, 2(1), 125-130.

18. Sidana V., Pasricha N., Makkar M., Bhasin S.: Group function occlusion, Indian J Oral Sci., 2012, 3: 124-128.
19. Panigrahi D., Satpathy A., Patil A., Patel G.: Occlusion and occlusal indicating materials, International Journal of Applied Dental Sciences, 2015: 1(4): 23-26.

20. Koos B., Godt A., Schille C., Göz G.: Precision of an instrumentation-based method of analyzing occlusion and its resulting distribution of forces in the dental arch, J. Orofac Orthop., 2010, 71:403-410.

21. Taruna M., Chittaranjan B., Sudheer N. ,Tella S., Abusaad Md.: Prosthodontic Perspective to All-On-4® Concept for Dental Implants, J. of Clinical and Diagnostic Research, $2014,8(10): 16-19$.

22. Parnia F., Moslehi E., Sadar K., Motiagheny N.: Pattern of occlusal contacts in eccentric mandibular position in dental students, J. Dent Res Dent Clin Dent Prospect, 2008 , 2:85-9.

23. Shirani M, Mosharraf R, Shirany M. Comparisons of patient satisfaction levels with complete dentures of different occlusions: a randomized clinical trial. J Prosthodont. 2014;23:259-266.

24. Jemt T., Lundquist S., Hedegard B.: Group function or canine protection, J. Prosthet Dent, 1982, 48:719-24. 